\title{
Scaled-up preparation of drug-loaded electrospun polymer fibres and investigation of their continuous processing to tablet form
}

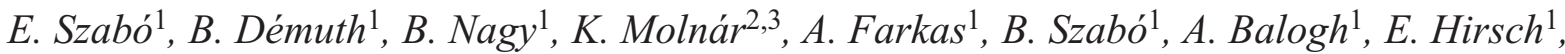 \\ B. Nagy ${ }^{4}$, G. Marosi ${ }^{1 *}$, Z. K. Nagy ${ }^{1 *}$ \\ ${ }^{1}$ Department of Organic Chemistry and Technology, Faculty of Chemical Technology and Biotechnology, Budapest \\ University of Technology and Economics, H-1111 Budapest, Müegyetem rkp. 3., Hungary \\ ${ }^{2}$ Department of Polymer Engineering, Faculty of Mechanical Engineering, Budapest University of Technology and \\ Economics, H-1111 Budapest, Müegyetem rkp. 3., Hungary \\ ${ }^{3}$ MTA-BME Research Group for Composite Science and Technology, Müegyetem rkp. 3, H-1111 Budapest, Hungary \\ ${ }^{4}$ Quick 2000 Ltd, H-4400 Tiszavasvári, Kabay J. u. 29., Hungary
}

Received 3 November 2017; accepted in revised form 19 December 2017

\begin{abstract}
Polymer-based electrospun amorphous solid dispersions (ASDs) were prepared and investigated from pharmaceutical application point of view. Spironolactone (SPIR) was used as model drug mixed in various concentrations with polymers suitable for fibre formation, such as vinylpyrrolidone-vinyl acetate copolymer, polyvinylpyrrolidone K30 and hydroxypropyl methylcellulose. Single needle electrospinning was applied at first for screening the composition of the prepared ASDs. Scaling-up the selected polymer-drug combination was accomplished by high speed electrospinning, the productivity of which enabled investigation of downstream processing to generate tablet formulation. The steps of a potential continuous production line (fibre collection, grinding, feeding and tableting) proved to be feasible with the electrospun ASD without any sign of crystallization. If crystalline drug was added into the ASD containing tablets as impurity strictly monotonous decrease of drug dissolution was observed in the function of the crystalline drug content. The capabilities of the non-destructive Raman and near-infrared spectroscopies, as fast quality assurance tools, were compared to each other in quantifying of crystalline SPIR content in the prepared tablets.
\end{abstract}

Keywords: industrial applications, high speed electrospinning, amorphous solid dispersions, tableting, continuous processing technologies

\section{Introduction}

Nowadays, significant part of active pharmaceutical ingredients (APIs) belongs to Class II of the Biopharmaceutical Classification System [1], that means these drugs possess low solubility and high permeability. By increasing the dissolution rate and extent of these drugs their inadequate bioavailability can be enhanced [2]. Polymers of high water solubility can form amorphous solid dispersions (ASDs) with such drugs improving their dissolution characteristics because there is no need of energy to break the crystal lattice structure [3]. Further advantages, such as reduced particle size, improved wettability and higher porosity, are also characteristic to ASDs [4]. That is why, this kind of formulation is getting to play a more and more important role in the pharmaceutical developments [5]. Among several melting, solvent and solvent-melting methods published for preparing ASDs [6], electrospinning (ES) capable of producing fibrous structures of large surface area seems to be

$\overline{{ }^{*} \text { Corresponding author, e-mail: zsknagy@,oct.bme.hu }}$ (C) BME-PT 
especially favourable in the field of pharmaceutical application [7-18].

Although the electrospinning (ES) is fast developing with appearance of tri-axial, coaxial, side-by-side and their combination processes [19-22] the most common and facile ES equipment is the single needle apparatus [23, 24]. The single needle electrospinning (SNES) applies a syringe filled with a polymerdrug containing solution, which is then forced through a nozzle attached to high voltage. However, the productivity of SNES does not satisfy the pharmaceutical requirements thus scaling-up of the technology is essential. In recent years, many methods have been created for this purpose such as needleless electrospinning [25-28], multineedle electrospinning [29], alternating current electrospinning [30-32], and high-speed electrospinning (HSES) [33]. The latter one, where electrostatic and centrifugal forces are combined, may be a highly suitable technology in the pharmaceutical industry for mass production of fibrous drug delivery systems.

The inherently continuous nature of ES can be considered as a great advantage. This advantage should even be enhanced by coupling the production of fibres with continuous downstream processing. HSES device coupled with continuous fibre collection might be utilized as a part of a continuous processing line. It is a well-known fact that continuous manufacturing is superior to batch processing owing to its cost effectiveness, robustness, better product quality control, and so on. Furthermore, continuous manufacturing meets the requirements of environmental protection and it can be more economical than a batch processing [34, 35]. ES can perfectly fit into this perspective. However, there are some cornerstones, such as continuous collection, grinding and feeding of the fibres, which cannot be neglected if continuous downstream processing is desired to be applied.

Utilization of the potential advantages of continuous technologies requires real-time process monitoring. Thus the pharmaceutical industry is increasingly focusing on process analytical technologies (PAT), which means basically, a large number of analytical tools, such as Raman, near infrared (NIR) or UV spectrometers, monitoring the products and processes $[36,37]$. These methods have been applied successfully as in-line techniques in many cases. For instance, NIR spectroscopy proved to be appropriate for examining the blend uniformity, content uniformity, and the tablets coating thickness $[38,39]$. Utilization of Raman spectroscopy also seems to be feasible for real-time monitoring of freeze-drying [40] and even real-time feedback control of continuous powder blending [41]. Furthermore, a PAT system can be particularly important and useful when tablets include ASDs since these metastable systems tend to crystallize during processing [42-44]. Raman and NIR spectroscopies can allow the detection of crystalline traces in the ASDs $[45,46]$, thus these analytical methods can be of enormous significance for in-line process monitoring [47].

Our goal with this work was to achieve scaled-up production of spironolactone (SPIR) containing electrospun ASD after optimization of polymers matrices and drug loadings. Fibrous ASD produced on a larger scale were intended to be formed as immediaterelease tablets. It was of interest whether continuous manufacturing steps such as continuous fibre collection, grinding, feeding, and tableting can be realized with the so-obtained electrospun ASD. Ultimately, the quantity of crystalline API was needed to be detected by Raman and NIR spectroscopy, while these two techniques were also compared. As the best knowledge of the authors, preparation of electrospun ASDs containing tablets by investigation of continuous process steps has never realized before. The whole investigation was intended to serve as a basis of continuous downstream processing of fibrous ASDs to prepare immediate-release tablets.

\section{Experimental}

\subsection{Materials}

Spironolactone (SPIR) with a molar weight of $417 \mathrm{~g} / \mathrm{mol}$, a melting point of $205-220^{\circ} \mathrm{C}$ and a water solubility of $28 \mathrm{mg} / \mathrm{L}$ [48] was kindly provided by Gedeon Richter Plc. (Budapest, Hungary). PVPVA64 (Kollidon ${ }^{\circledR}$ VA64) obtained from BASF (Ludwigshafen, Germany) is a vinylpyrrolidone-vinylacetate amorphous copolymer (6:4) with a molecular weight in the range of 45-70 kDa. Hydroxypropyl methylcellulose (HPMC) $29105 \mathrm{mPa} \cdot \mathrm{s}$ was supplied by Aqualon, Hercules (Zwijndrecht, the Netherlands). Polyvinylpyrrolidone K30 (PVPK30, Plasdone ${ }^{\circledR}$ K30) was provided by Ashland Inc. (Schaffhausen, Switzerland). Microcrystalline cellulose (MCC, Vivapur ${ }^{\circledR}$ 200) was purchased from JRS Pharma (Rosenberg, Germany). Lactose (Tablettose ${ }^{\circledR} 80$ ) was supplied by Meggle Pharma (Wasserburg, Germany). Croscarmellose sodium (Ac-Di-Sol ${ }^{\circledR} \mathrm{SD}-711$ ) was a kind gift from FMC BioPolymer (Brussels, Belgium). All other 
excipients and solvents were analytical grade and used without further purification.

\subsection{Single-needle electrospinning (SNES)}

The electrostatic spinneret (with an inner diameter of $0.5 \mathrm{~mm}$ ) applied for the experiments were connected with an NT-35 high voltage DC supply (MA2000; Unitronik Ltd, Nagykanizsa, Hungary). The electrical potential applied on the spinneret electrode was adjusted during the experiments to $35 \mathrm{kV}$. A grounded aluminium plate covered with aluminium foil was used as collector. The distance of the spinneret and the collector was set to $15 \mathrm{~cm}$. The experiments were performed at room temperature $\left(25^{\circ} \mathrm{C}\right)$ and at $45 \pm 5 \%$ relative humidity. Polymer solutions were dosed by a SEP-10S Plus type syringe pump (Aitecs, Vilnius, Lithuania). Preparation of ASDs with spironolactone and various polymers was accomplished by SNES. The dosing rate was $6 \mathrm{~mL} / \mathrm{h}$.

\subsection{High speed electrospinning (HSES) and collection, milling and feeding of the fibres}

The scaled-up electrospinning experiments were performed using a high speed electrostatic spinning setup (Quick2000 Ltd, Tiszavasvári, Hungary) consisting of a round-shaped, stainless steel spinneret connected to a high speed motor [33]. The polymer solution was fed with a peristaltic pump with a flow rate of $200 \mathrm{~mL} / \mathrm{h}$. The rotational speed of the spinneret was fixed at $15000 \mathrm{rpm}$, and the voltage applied on it was $40 \mathrm{kV}$ during the experiments. The grounded collector covered with aluminium foil was placed $30 \mathrm{~cm}$ from the spinneret in all cases. The experiments were performed at ambient temperature $\left(25^{\circ} \mathrm{C}\right)$ and at $45 \pm 5 \%$ relative humidity.

To insert ES into continuous manufacturing lines, collection and forwarding of the fibres to the milling equipment, and then the grinding step itself have to be solved. A piece of equipment designed and built by the authors for continuous collection is described in Section 3.2.4. The electrospun material was pushed through a sieve with hole size of $0.8 \mathrm{~mm}$ to make it suitable for blending with excipients. This kind of milling corresponds to oscillatory or conical milling that can be utilized in continuous manufacturing. The ability to feed the material into hoppers is also inevitable. Feeding experiments were performed on a DDW-MD0-MT type, twin-screw loss-in-weight feeder (Brabender Technologie (Duisburg, Germany). The diameter of the screws was $12 \mathrm{~mm}$.

\subsection{Differential scanning calorimetry (DSC)}

DSC measurements were performed by a Setaram DSC 92 (Calure, France) apparatus (sample weight: $\sim 10-15 \mathrm{mg}$, open pan, nitrogen flush, $50 \mathrm{~mL} / \mathrm{min}$, 1.6 bar). The temperature program consisted of an isothermal part of $1 \mathrm{~min}$ at ambient temperature and subsequent linear heating from 25 to $250^{\circ} \mathrm{C}$ with a rate of $10^{\circ} \mathrm{C} / \mathrm{min}$.

\subsection{Thermogravimetric analysis (TGA)}

Thermogravimetric examination of the selected composition was accomplished by a Q5000 TGA instrument (TA Instruments, USA). The sample was heated up from 25 to $300^{\circ} \mathrm{C}$ by $10^{\circ} \mathrm{C} / \mathrm{min}$. The applied nitrogen flush was $50 \mathrm{~mL} / \mathrm{min}$ during the measurement.

\subsection{X-ray powder diffraction (XRPD)}

$\mathrm{X}$-ray powder diffraction patterns were docketed with a PANalytical X'pert Pro MDP X-ray diffractometer (Almelo, The Netherlands) using $\mathrm{Cu}-\mathrm{Ka}$ radiation $(1.506 \AA)$ and Ni filter. The applied voltage was $40 \mathrm{kV}$, while the current was $30 \mathrm{~mA}$. The untreated materials and the fibrous samples were analysed between $2 \theta$ angles of 4 and $44^{\circ}$.

\subsection{Scanning electron microscopy (SEM)}

Morphology of the samples was examined by a JEOL 6380LVa (JEOL, Tokyo, Japan) type scanning electron microscope in high vacuum. Samples were fixed by conductive double-sided carbon adhesive tape and sputtered by gold using ion sputter (JEOL 1200, JEOL, Tokyo, Japan). Applied accelerating voltage was set between 5 and $15 \mathrm{kV}$ and working distance was $10 \mathrm{~mm}$.

\subsection{In vitro dissolution measurement}

Dissolution studies were performed on a Pharmatest PTWS 600 dissolution tester (Pharma Test Apparatebau AG, Hainburg, Germany). In case of the pure ASDs the basket method (United States Pharmacopoeia I) was used, while the tablets were examined by paddle method (United States Pharmacopoeia II). Samples equivalent to $50 \mathrm{mg}$ of spironolactone were weighed without any pre-treatment into the dissolution baskets and immersed in $900 \mathrm{~mL}$ of $0.1 \mathrm{~N} \mathrm{HCl}$ dissolution medium kept at a constant temperature of $(37 \pm 0.5){ }^{\circ} \mathrm{C}$ and stirred at $100 \mathrm{rpm}$. An on-line coupled Agilent 8453 UV-Vis spectrophotometer (Hewlett-Packard, Palo Alto, USA) was used to measure the concentration of dissolved spironolactone at 
a wavelength of $243 \mathrm{~nm}$. Based on a preliminarily built calibration (from 1 to $50 \mathrm{mg} / \mathrm{L}$ ), the concentration of the dissolved SPIR could be readily calculated during the measurement. All of the dissolution measurements were accomplished triplicates in case of each composition and sample.

\subsection{Measurement of the drug solubility}

The amount of the dissolved drug was determined by using UV-Vis spectroscopy. $8.3 \mathrm{mg}$ polymer and $11.1 \mathrm{mg}$ SPIR were weighed in a $100 \mathrm{~mL}$ flask filled up subsequently with the acidic $(\mathrm{pH}=1)$ medium. The weight of the drug and the polymer was calculated proportionally based on the dissolution measurements where $125 \mathrm{mg} \mathrm{40 \%} \mathrm{SPIR} \mathrm{containing} \mathrm{ES}$ product was applied in $900 \mathrm{~mL}$ dissolution medium. The solutions were stirred for 2 days on a magnetic stirrer. Before the UV-Vis measurement, the solutions were filtered through a $0.45 \mu \mathrm{m}$ filter. Calculation of the concentration was performed similarly to the in vitro dissolution test.

\subsection{Powder characterization and tableting}

Bulk and tapped densities of powder mixture for tableting were examined on an ERWEKA SVM12 (Heusenstamm, Germany) type tapped density tester. Hausner ratio can be calculated based on Equation (1) [49] and Carr index [50] may be determined according to the Equation (2):

$$
\begin{aligned}
& \text { Hausner ratio }=\frac{\rho_{\text {tapped }}}{\rho_{\text {bulk }}} \\
& \text { Carr idex }=100 \cdot\left(\frac{\rho_{\text {tapped }}-\rho_{\text {bulk }}}{\rho_{\text {trapped }}}\right)
\end{aligned}
$$

The tableting was performed using a Dott Bonapace CPR6 eccentric tableting machine (Limbiate, Italy) equipped with $14 \mathrm{~mm}$ concave punches. The applied compression force was about $10 \mathrm{kN}$. For the dissolution measurement, $625 \mathrm{mg}$ tablets were prepared in which the API was $50 \mathrm{mg}$ (125 mg ASD) and they also contained $10 \%$ disintegrant (Ac-Di-Sol ${ }^{\circledR}$ ), 1\% glidant (Aerosil), $1 \%$ lubricant (magnesium stearate), and $34-34 \%$ tablet fillers (MCC, lactose). Tablet breaking force (with 10 tablets) was measured on a Schleuniger 4M type hardness tester (Thun, Switzerland).

\subsection{Raman spectroscopy}

One of the spectroscopy methods for detection of crystalline API in blends and tablets was the Raman spectroscopy. A Kaiser RamanRxn $2{ }^{\circledR}$ Hybrid in situ analyzer (Kaiser Optical Systems, Ann Arbor, USA) connected with PhAT (Pharmaceutical Area Testing) probe was applied to gain the spectra of the samples. A $400 \mathrm{~mW}, 785 \mathrm{~nm}$ diode laser (Invictus) was utilized for illuminating the products. Reflection mode was used for measuring calibration samples of powder mixtures and tablets. In addition, the device was also operated in transmission mode in case of tablets. The diameter of the laser spot size was extended to $6 \mathrm{~mm}$ and the nominal focus length was $250 \mathrm{~mm}$. The examined spectral range was between 200 and $1890 \mathrm{~cm}^{-1}$. During the measurements the resolution was $4 \mathrm{~cm}^{-1}$, which provided 1690 variables during data processing. In case of reflection mode, an acquisition time of 30 seconds gave adequate Raman intensity and signal-to-noise ratio. In transmission mode, acquisition time of 2 minutes was adjusted due to $4 \mathrm{~mm}$ thickness of $625 \mathrm{mg}$ tablets to reach similar quality with the backscattered spectra. 5 spectra per powder samples were acquired and 2 repeated measurements per tablets (both side of the products) were satisfactory for calibration.

\subsection{NIR spectroscopy}

The other applied spectroscopy instrument was a Fourier-transform Near Infrared (FT-NIR) spectrometer (Bruker MPA NIR, Bruker OPTIK GmbH, Ettlingen, Germany) in diffuse reflection mode. Spectra were obtained in the spectral range of 3700 $15000 \mathrm{~cm}^{-1}$ with a resolution of $8 \mathrm{~cm}^{-1}$, and 128 scans were accumulated. A high intensity NIR light source (Tungsten) was utilized as excimer, and the $\mathrm{PbS}$ detector operated at room temperature. Double Sided Forward-Backward acquisition mode was used during the measurements. Simple glass and tablet sample holders were used for investigating powder mixtures and tablets. Number of the repetitions was the same as in Raman measurements. However, the experiments were taken place only in reflection mode because transmission method was not possible due to the tablet thickness.

\subsection{Multivariate calibration of Raman and NIR spectroscopy}

Calibration samples of $2.5 \mathrm{~g}$ powder were prepared for Raman and NIR spectroscopic studies with 0 , $0.5,2,5,10,20,50$ and $100 \%$ crystalline spironolactone content followed by preparation of three tablets of $625 \mathrm{mg}$ at each concentration levels. Both 
Raman and NIR spectroscopy measurements were evaluated by using MATLAB 8.2. (MathWorks, USA) program with PLS Toolbox 7.8.2. (Eigenvector Research, USA). Partial least squares (PLS) regression method was applied for the determination of the crystalline API content. During PLS modelling coordinate transformation was performed on the original $n \times \lambda$ sized dataset (where $n$ is the number of spectra used for the calibration and $\lambda$ is the number of variables, i.e. wavenumbers). The created new variables (latent variables, LVs) combine the original variables in the way that the latent variables are orthogonal to each other, and the first few variables describe the possible highest covariance with the dependant variable (in this case the crystalline API content). The number of latent variables, necessary for the model, was determined by minimizing the root mean square error of cross validation (RMSECV).

The performance of the PLS model can be further improved by selecting a subset of spectrum wavenumbers resulting in the most accurate model. For this, interval PLS variable selection method was applied [51], which is a systematic search for the best combination of variables. The spectra are divided into variable windows (consecutive variable subsets) sized depending on user requirement (in this study 50 wavenumbers/windows were used), then several PLS models are built with systematically adding (forward mode) or eliminating (reverse mode) variable windows for the calculations. The searching algorithm is running until the model performance (e.g. RMSECV) cannot be improved by including or excluding additional variable windows.

All spectra were pre-processed before PLS modelling. In the case of Raman spectra, Automatic Whittaker Filter baseline correction with asymmetry parameter $\mathrm{p}=0.001$ and smoothing parameter $\lambda=10^{5}$, normalization to unit length, and mean centring were applied. NIR spectra were corrected by SavitzkyGolay first derivative (15 points/window, second order smoothing), standard normal variate (SNV), and mean centring. The models were validated by $6-$ fold venetian blind cross-validation leaving out one sample per concentration levels at each calculation step. The models were compared by the coefficient of determination $\left(R^{2}\right)$, the root mean square error of calibration and cross-validation (RMSEC/RMSECV) and by the limit of detection (LoD) and limit of quantification (LoQ).

\section{Results and discussion}

SPIR containing ASDs were prepared by SNES and HSES for enhancing the drug dissolution. Three different polymers (HPMC, PVPK30 and PVPVA64) and three different drug loadings (20,30, and 40\%) were screened by SNES. The best composition was chosen based on the in vitro dissolution tests. Scaledup production of this composition was performed using HSES. Furthermore, parts of a possible continuous line (collection, grinding, feeding) were tested as well. Eventually, the effect of crystalline API content on the dissolution of tablets containing electrospun ASD was investigated. Raman and NIR spectroscopy was applied for detection of crystalline and amorphous forms of SPIR and the performance of these two methods were compared.

\subsection{Screening study by SNES}

Limited data is available on the electrospun SPIR containing ASDs. As a consequence, a screening study with three polymers and optimization of polymer-drug concentration was to be done at first. Table 1 presents the different polymer-drug combinations investigated.

HPMC requires the application of DCM, and only small concentration of it can be achieved in the solution. However, with the vinylpyrrolidone-based polymers (PVPK30 and PVPVA64), more environmentally friendly solvent combinations were attempted to be used. The utilization of IPA is particularly interesting since this is not a conventional ES solvent. Nevertheless, the fibre formation was feasible for each composition.

\subsubsection{DSC and XRPD results}

The amorphicity of the different compositions was examined by DSC. Based on the thermograms obtained (Figure 1a), it can be concluded that the melting peak of the drug $\left(\sim 211^{\circ} \mathrm{C}\right)$ did not appear for the electrospun products. This indicates that spironolactone was amorphous in all these compositions. Appearance of peaks between 65 and $94.4^{\circ} \mathrm{C}$ can be explained by the presence of water absorbed by the ASD. Peaks between 222 and $240^{\circ} \mathrm{C}$ indicate the decomposition of SPIR. Interestingly, HPMC mitigate this decomposition as a significantly smaller peak appears in the resulted thermograms. Total amorphisation is also supported by results of XRPD: the sharp peaks of crystalline SPIR did not appear with any of the different fibrous samples (Figure 1b). 
Table 1. Various composition of electrospun solutions and dissolution results of the ES products. Applied dissolution method: $900 \mathrm{~mL} 0.1 \mathrm{M} \mathrm{HCl}$ dissolution medium, $37 \pm 0.5^{\circ} \mathrm{C}, 100 \mathrm{rpm}$, basket method, $50 \mathrm{mg}$ API content. (DCM: dichloromethane; EtOH: ethanol; ACE: acetone; IPA: isopropyl alcohol; bold: chosen composition for scale-up).

\begin{tabular}{|l|c|c|c|c|c|}
\hline \multicolumn{1}{|c|}{ Samples } & $\begin{array}{c}\text { Polymer } \\
{[\mathbf{m g}]}\end{array}$ & $\begin{array}{c}\text { Spironolactone } \\
{[\mathbf{m g}]}\end{array}$ & $\begin{array}{c}\text { Applied Solvents } \\
{[\mathbf{8} \mathbf{~ m L}]}\end{array}$ & \multicolumn{2}{c|}{$\begin{array}{c}\text { Dissolved drug at 5 and 90 min } \\
{[\mathbf{\%}]}\end{array}$} \\
\hline HPMC_SPIR20\% & 600 & 150 & DCM+EtOH $(1: 1)$ & 28.0 & 83.8 \\
\hline HPMC_SPIR30\% & 600 & 257 & DCM+EtOH $(1: 1)$ & 28.4 & 81.4 \\
\hline HPMC_SPIR40\% & 600 & 400 & DCM+EtOH $(1: 1)$ & 22.1 & 67.9 \\
\hline PVPK30_SPIR20\% & 2000 & 500 & ACE+EtOH $(1: 1)$ & 8.9 & 50.8 \\
\hline PVPK30_SPIR30\% & 2000 & 857 & ACE+EtOH $(1: 1)$ & 11.1 & 48.3 \\
\hline PVPK30_SPIR40\% & 2000 & 1333 & ACE+EtOH $(1: 1)$ & 16.3 & 49.2 \\
\hline PVPVA64_SPIR20\% & 2100 & 525 & IPA+ACE $(2: 1)$ & 41.1 & 68.5 \\
\hline PVPVA64_SPIR30\% & 2100 & 900 & IPA+ACE $(2: 1)$ & 74.2 & 79.7 \\
\hline PVPVA64_SPIR40\% & $\mathbf{2 1 0 0}$ & $\mathbf{1 4 0 0}$ & IPA+ACE (2:1) & $\mathbf{7 8 . 9}$ & $\mathbf{8 7 . 9}$ \\
\hline cryst. SPIR & $\mathrm{n} / \mathrm{a}$ & $\mathrm{n} / \mathrm{a}$ & $\mathrm{n} / \mathrm{a}$ & 4.7 & 17.5 \\
\hline
\end{tabular}
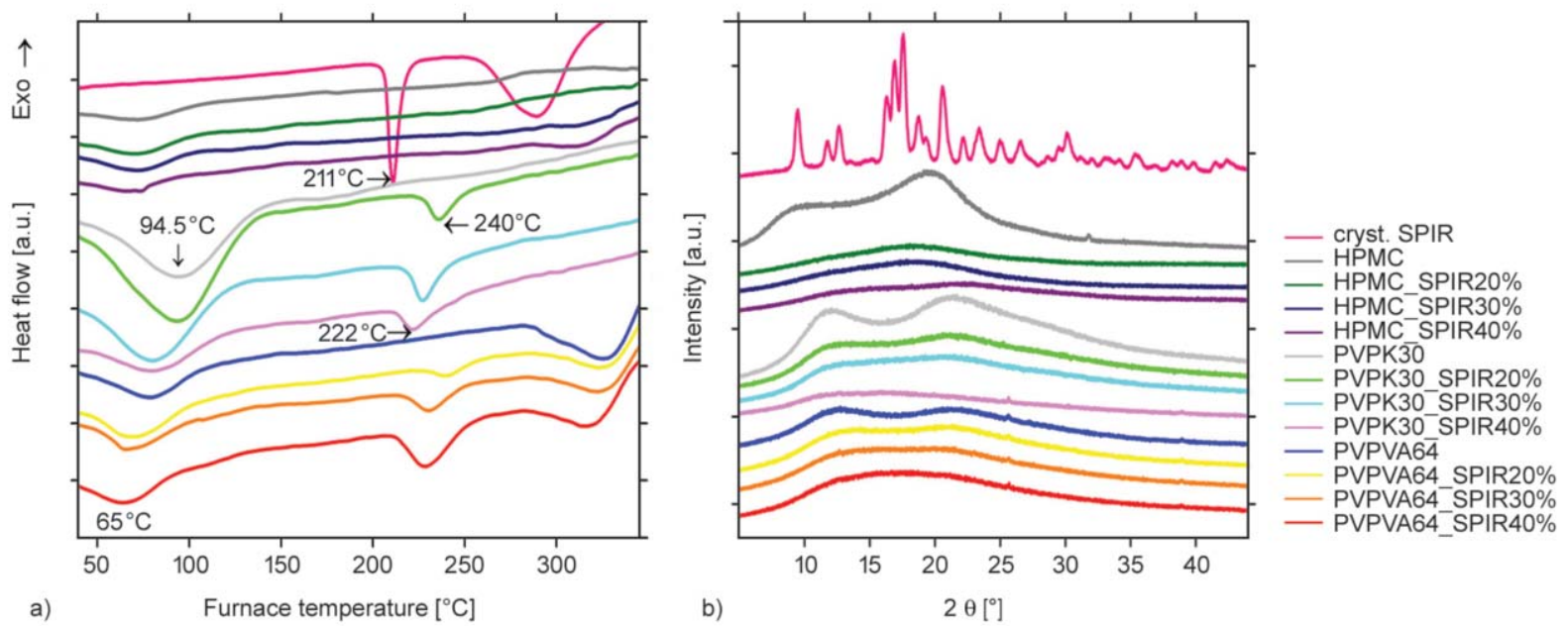

Figure 1. Results of DSC (a) and XRPD (b) experiments.

\subsubsection{Selection of the optimal composition based on the dissolution}

According to the dissolution results (Table 1), the well water-soluble PVPVA64 provided the best dissolution profile since nearly $80 \%$ of SPIR was released in the first 5 minutes from the PVPVA64_SPIR40\% sample. Slower dissolutions and lower extents were observed with HPMC systems (less than 30\% drug dissolved in 5 minutes). PVPK30 gave the least favourable result: approximately only two times more drug was released at 90 minutes than in the case of the crystalline drug. The amount of the SPIR molecules dissolved from the crystalline sample into the medium was $17.5 \%$ at 90 minutes.

Not only the higher dissolution, but the extent of supersaturation is of great interest since it can determine the diffusion performance of the drug through membranes [52]. The solubility of SPIR, both in pure form and mixed with polymers, was determined in $0.1 \mathrm{~N} \mathrm{HCl}$ by UV-Vis spectroscopy after 2 days of stirring at room temperature. Three repeated measurements were performed in all cases. It can be established that polymers increase the solubility of crystalline SPIR slightly, but there is no significant difference between them (Figure 2).

High polymer content of the tablet can, however, generate disadvantageous tablet properties such as slow disintegration. Therefore, the polymer content should be reduced as much as possible. Based on these considerations and on the obtained results the ASD of PVPVA64 and $40 \%$ SPIR seemed to be the most suitable for further experiments.

\subsubsection{Investigation of the fibres with SEM}

The selected composition was investigated by SEM to get information on the sample morphology. Electrospinning is well-known for its capability of producing nanofibres with enhanced surface area with 


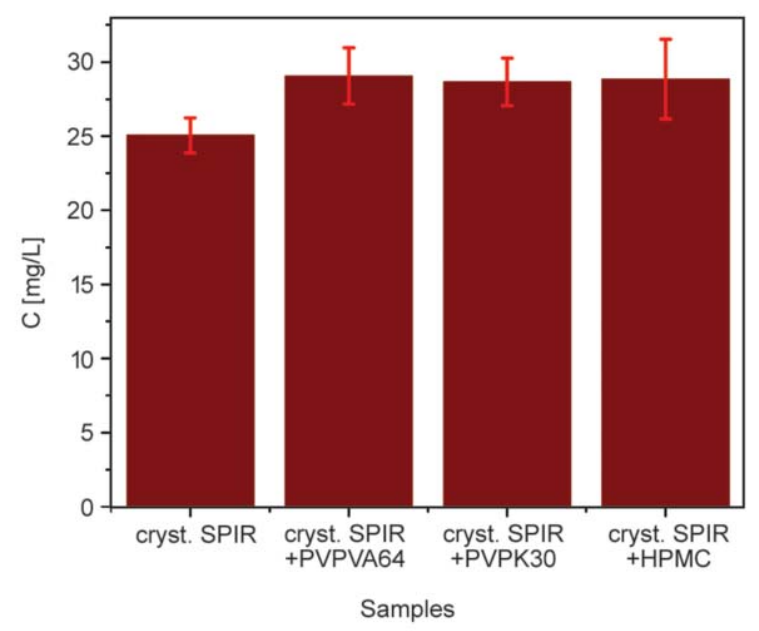

Figure 2. Solubility of different polymer-drug mixtures $(n=3)$.

which instantaneous release can be achieved $[9,10$, 53]. Fibres with diameter in the nano range could be produced with PVPK30 and HPMC polymers (data not shown). With IPA, a solvent with relatively large viscosity $\left(2.86 \mathrm{cP}\right.$ at $\left.15^{\circ} \mathrm{C}\right)$, slightly thicker PVPVA64based fibres with some beads could be obtained (Figure 3a). An increase in viscosity usually leads to thicker fibres [54, 55]. However, it can occur that fibres with larger diameter release the encapsulated drug faster than nanofibres due to the easier accessibility [56]. Based on the SEM images, it can be stated that the majority of the fibres prepared by SNES possesses a diameter below $5 \mu \mathrm{m}(4.26 \pm 2.08 \mu \mathrm{m})$, therefore their specific surface is fairly large. As it is shown hereinafter, the dissolution rate was not impaired, fast release could be achieved. Another interesting fact is the fragmentation of the fibres which can cause by PVPVA64. For this reason, a comparison was made between the SEM images of the fibre

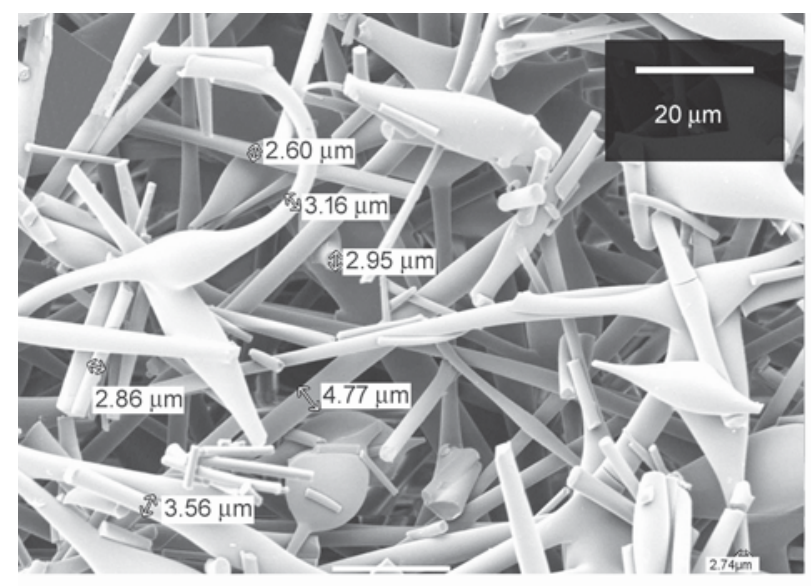

a) before grinding (Figure 3a) and after grinding (Figure $3 b$ ). It can be seen in Figure 3 that no significant differences are between the two images. This observation proves that the fibres are very fragile, which is advantageous from the downstream processing (e.g. grinding) point of view.

\subsection{Scaling-up of the electrospinning}

SNES samples had appropriate dissolution and morphology, but SNES is known to be inappropriate for mass production of the fibres. The technology of HSES was used to increase the productivity. The scaled-up electrospinning technique was applied with the optimal composition (matrix: PVPVA64, drug loading: $40 \%$ ) and more than thirtyfold productivity was achieved during this production (SNES: $\sim 2.1 \mathrm{~g} / \mathrm{h}$; HSES: $\sim 70 \mathrm{~g} / \mathrm{h}$ ). In consequence, the amount of the ES material produced by HSES was sufficient for examining tableting and continuous manufacturing circumstances.

\subsubsection{DSC, XRPD and SEM results of the HSES sample}

Characterization of the product was investigated by the methods used previously, such as DSC, XRPD and SEM. Figures $4 a, b$ prove the success of scaling-up: total amorphisation of SPIR was achieved for this ASD as well (according to DSC and XRPD results). The same peak has been appeared in case of HSES samples in the DSC results than before by the SNES materials. For this reason, a TGA measurement was performed with the selected composition (Figure 5). A significant weight loss from the temperature of the endothermic peak $\left(\sim 200^{\circ} \mathrm{C}\right)$ can be seen in the

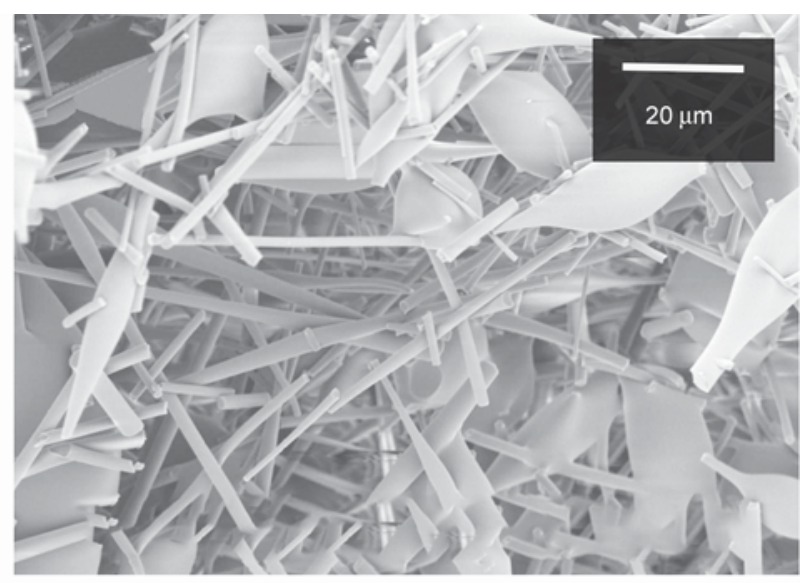

b)

Figure 3. Scanning electron microscopic images of the selected composition prepared by SNES before grinding (a) and after grinding (b) (PVPVA64_SPIR40\%). 


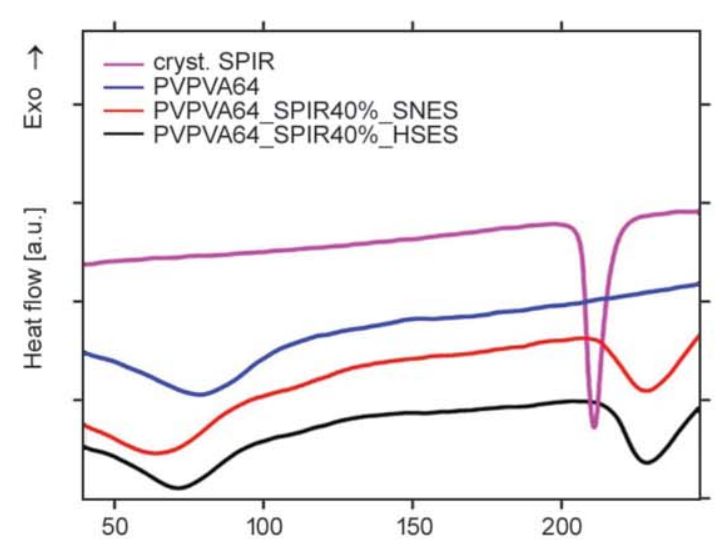

a)

Furnace temperature $\left[{ }^{\circ} \mathrm{C}\right]$

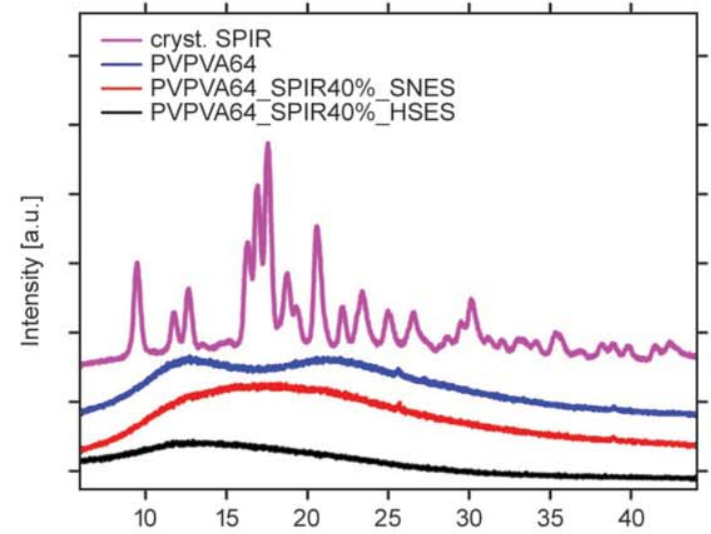

b)

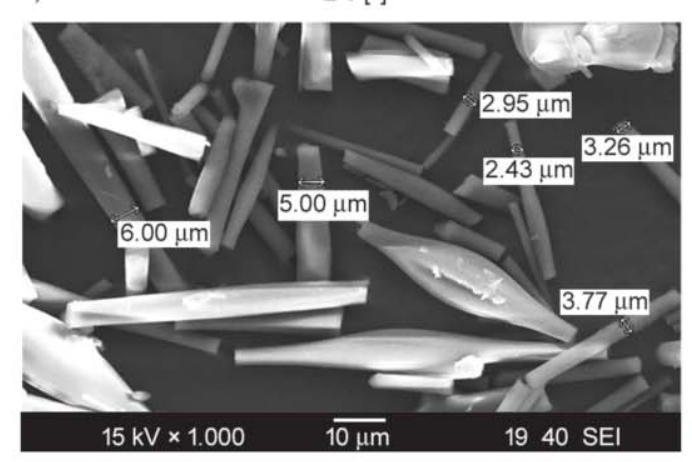

c)

Figure 4. Characterization of the fibrous ASD obtained by HSES by (a) DSC (b) XRPD and (c) SEM.

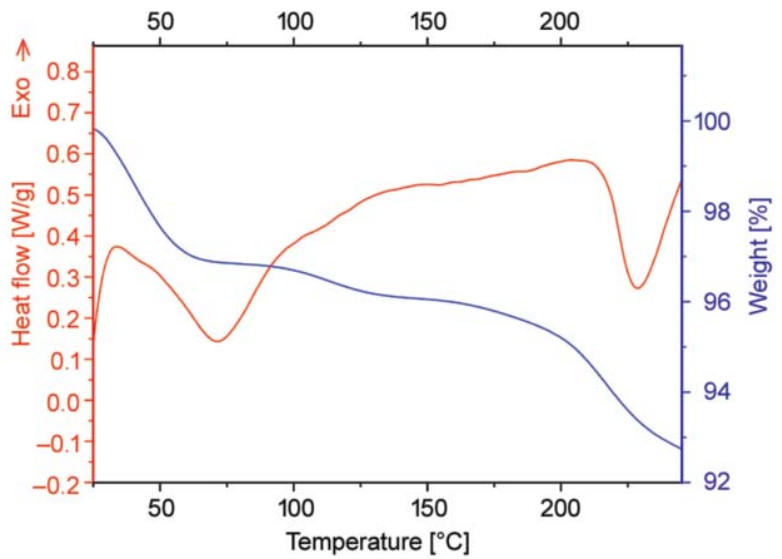

Figure 5. Comparison of the DSC and TGA results in case of the selected composition. thermogram which proves the decomposition of the SPIR. The morphology and the diameter of the HSES fibres $(5.70 \pm 3.75 \mu \mathrm{m})$, as shown in SEM image of the milled fibres (Figure 4c), were similar to the SNES materials. Grinding of the sample, which is an important step to obtain a blendable powder, is obviously noticeable on the image since appropriately short fibres could be obtained.

\subsubsection{In vitro dissolution of the HSES sample}

The dissolution of the HSES product was compared to that of the same SNES composition. Dissolution profiles were compared by the 'difference factor, $f_{1}$ ', and the 'similarity factor, $f_{2}$ ' $[57,58]$. These can be used in bioequivalence studies to compare the in vitro dissolutions of different forms. Based on our calculations $\left(f_{1}=0.39 \% ; f_{2}=98.14 \%\right)$, the dissolution characteristics of the HSES fibres were similar to the SNES materials which is also clearly visible in Figure 6.

\subsubsection{Tableting}

The next step was to convert the ASD into an applicable formulation. While the electrospun material has 'poor' flowability (according to the Hausner ratio: 1.36 and Carr index: 26\%), which might hinder the tableting process. Therefore, the addition of flowability aiding excipients is necessary. Interestingly, the powder mixture consisting of ASD of SPIR and PVPVA64 and the tableting excipients had much improved flowability, which is surprising considering results of similar subject in the literature [59]. (Hausner ratio: 1.12 , Carr index: $11 \%$; according to the

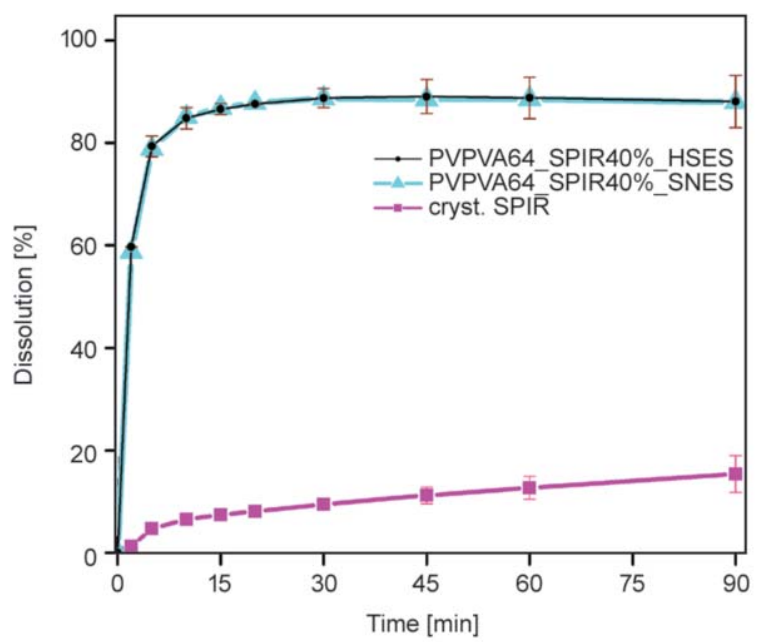

Figure 6. In vitro dissolution of the HSES product $(900 \mathrm{~mL}$ $0.1 \mathrm{M} \mathrm{HCl}$ dissolution medium, $37 \pm 0.5^{\circ} \mathrm{C}, 100 \mathrm{rpm}$, basket method, $50 \mathrm{mg}$ API content, $n=3$ ). 
Table 2. Physical properties of the powder mixture and the tablets.

\begin{tabular}{|l|l|l|}
\hline $\begin{array}{l}\text { Properties of the pow- } \\
\text { der mixture }\end{array}$ & Bulk density & $0.499 \mathrm{~g} / \mathrm{mL}$ \\
\cline { 2 - 3 } & Tapped density & $0.561 \mathrm{~g} / \mathrm{mL}$ \\
\cline { 2 - 3 } & Hausner Ratio & 1.12 \\
\cline { 2 - 3 } & Carr's index & $11 \%$ \\
\hline Properties of the tablets & Compression force & $10,72 \pm 1,47 \mathrm{kN}$ \\
\cline { 2 - 3 } & Weight & $625.9 \pm 0.8 \mathrm{mg}$ \\
\cline { 2 - 3 } & Tablet breaking force & $122.7 \pm 11.9 \mathrm{~N}$ \\
\hline & Friability & $0.23 \%$ \\
\hline & Thickness & $3.88 \pm 0.033 \mathrm{~mm}$ \\
\hline
\end{tabular}

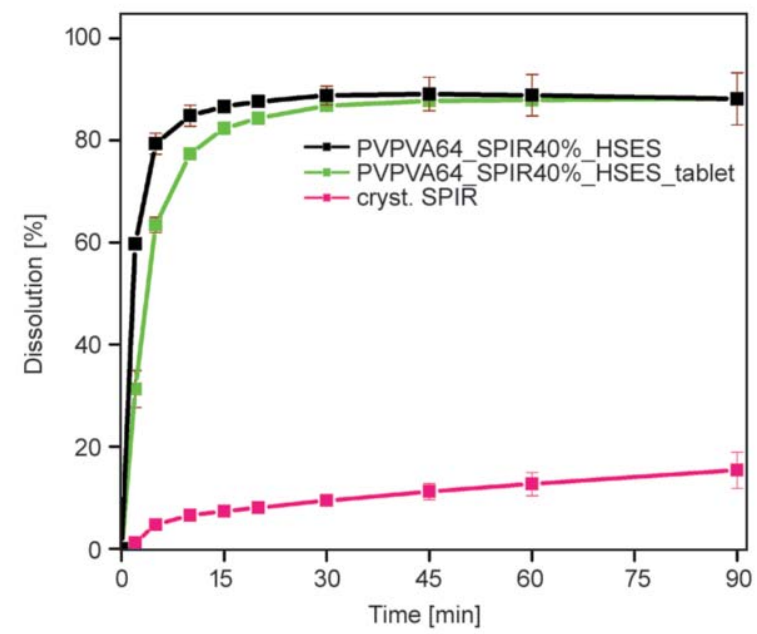

Figure 7. In vitro dissolution of amorphous spironolactone containing tablet $(900 \mathrm{~mL} 0.1 \mathrm{M} \mathrm{HCl}$ dissolution medium, $37 \pm 0.5^{\circ} \mathrm{C}, 100 \mathrm{rpm}$, basket method for HSES product and paddle method for tablets, $50 \mathrm{mg}$ API content, $n=3$ ).

U.S. Pharmacopeia, powder blends with Hausner ratios between 1.12 and 1.18 and Carr indices between 11 and $15 \%$ receive the second best, 'good' classification for flowability). The bulk density of this blend was also appropriate for tableting. The breaking force of the prepared tablets achieved high value between 100 and $134 \mathrm{~N}$ (Table 2).

The in vitro dissolution of the tablets was investigated in order to find out if ASD system retains its advantageous properties even after tableting. Figure 7 shows a comparison of dissolution profiles of crystalline SPIR, scaled-up ASD product, and tablet. Release of SPIR from the tablets was observed to have a similar extent and a slightly slower rate compared to that of the pure ASD.

\subsubsection{Insertion of ES into a continuous system}

Scaled-up production of drug-loaded fibres and preparation of appropriate blend and tablets has been proved to be feasible. One of the main pharmaceutical trends nowadays is the transfer from batch to continuous manufacturing. In order to reveal if ES fits into this perspective and can be inserted into continuous lines, some fundamentals have to be established. The possible way to generate tablets from electrospun ASDs in a continuous manner is depicted in Figure 8. The produced fibres need to be collected and forwarded to a milling equipment (milling was proven to be feasible earlier) and then to a lossin-weight feeder. After gravimetric feeding and homogenization, the tablets can obviously be produced in a continuous manner. Investigation of homogenization of fibrous ASD with excipients in a twinscrew blender and homogeneity is a subject of a different, ongoing study.

\subsubsection{Collection and milling of the fibres}

To achieve continuous production of electrospun fibre-based tablets, mechanical engineering developments are also needed. The first step is the continuous collection of the produced fibres, which is not solved in the pharmaceutical. For this purpose, we designed a machine made of aluminium with three rolls (Figure 9a, rolls are marked by 1, 2, and 3). The fibre collection occurs on a polypropylene (PP) fleece constantly moving on the rolls twirled by an electrical motor (12 V direct current motor, gear ratio 3000:1). This fleece is a hot pressed filament, which is strong, flexible, and thin enough not to have an effect on the electric field strength. One of the most important parameters is the distance between the spinneret and the grounded collector (marked by 4 in Figure 9a). In our experiment (PVPVA64 fibres), this was chosen to be $300 \mathrm{~mm}$, since this way the evaporation of the solvent can be complete, and sticking of the sheet to the fleece can be avoided. At the bottom of the equipment, a doctor blade made of aluminium peels the fibrous sheet off the fleece, making it nonwoven to be suitable for collecting fibres again (Figure 9b). This way the removed product could be easily forwarded to the next step, to the milling equipment (e.g. oscillational mill or conical mill, Figure 8). The milling of brittle PVPVA64-based fibres is feasible as it was shown earlier.

\subsubsection{Gravimetric feeding of the electrospun ASD}

In continuous manufacturing, the precise dosing of the components with loss-in-weight feeders is necessary to ensure satisfying tablet quality. However, low bulk density and weak flowability (like the fibrous 


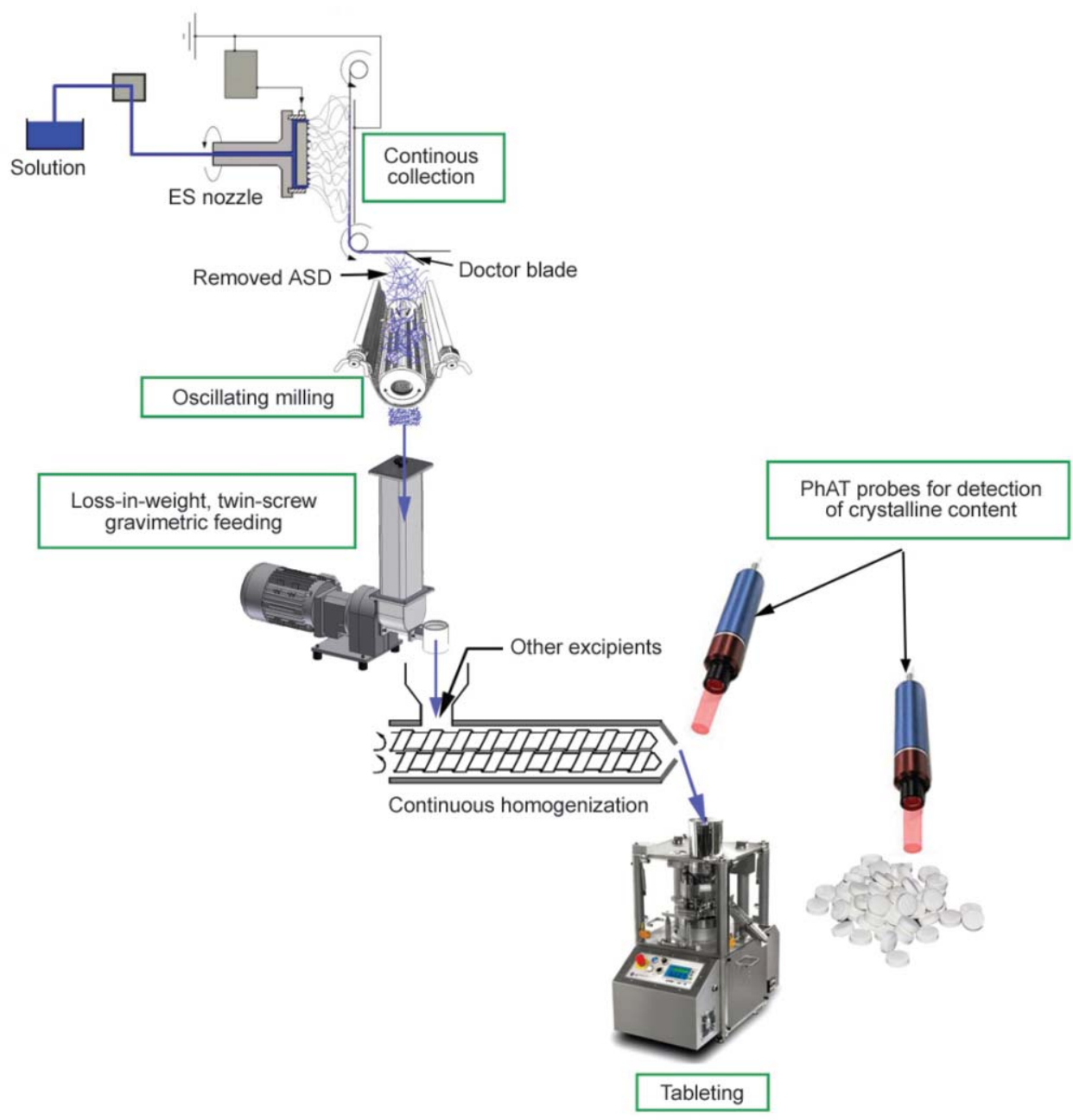

Figure 8. Schematic design of a continuous line from ES to directly compressed tablets with real-time detection of crystallinity. Green rectangles mark the areas investigated in this work.

ASD has) can hinder the appropriate feeding. The maximum mass rate for the fibrous ASD was $40 \mathrm{~g} / \mathrm{h}$ that could be maintained precisely by the twin screw gravimetric feeder with $12 \mathrm{~mm}$ screw diameter. Presumably, this rate can be easily enhanced by increasing the size of the feeder and/or the type of the screws. Another possible method to increase the feeding rate is the addition of feeding aiding excipients. In this case, MCC (Vivapur ${ }^{\circledR} 200$ ) was added to the fibres (double amount compared to the ASD), and $385 \mathrm{~g} / \mathrm{h}$

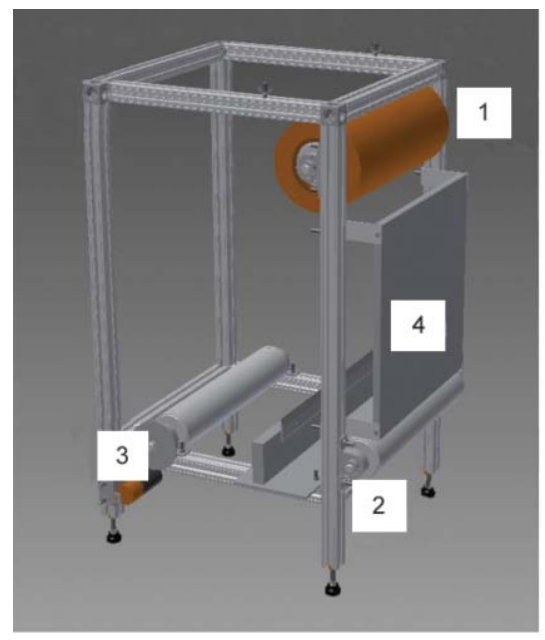

a)

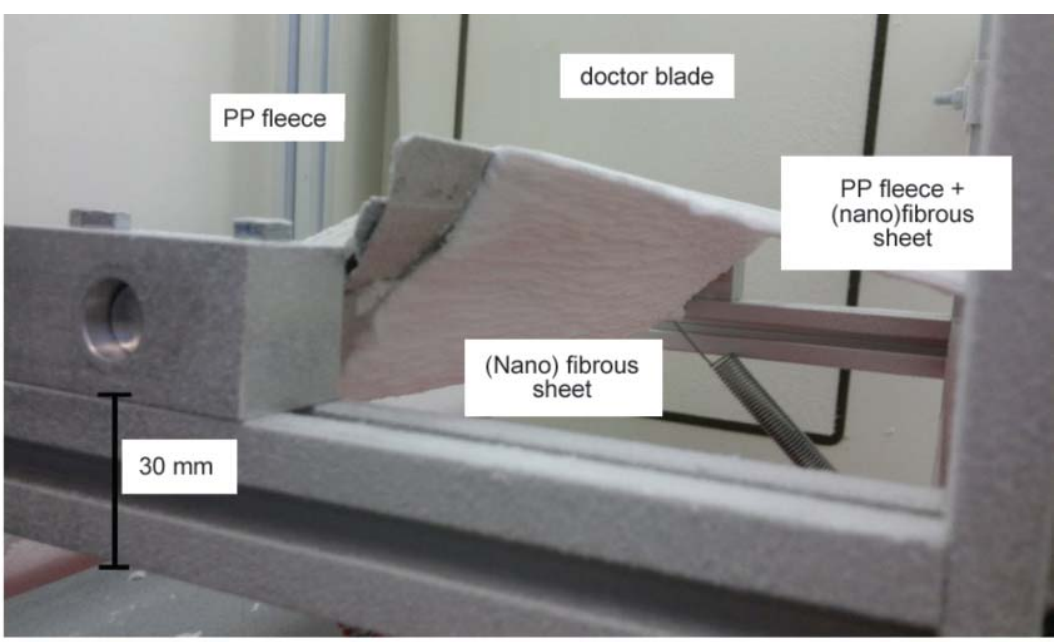

b)

Figure 9. Equipment for collecting fibres using by three rolls (a) and a blade (b) for peeling the samples. 
feeding rate was achieved. This means $\sim 130 \mathrm{~g} / \mathrm{h}$ for the electrospun ASD in case of 1:2 ES and MCC ratio, which is already a quite satisfying value for such a small system. At this scale 24000 tablets/day might be manufactured with $50 \mathrm{mg}$ dose of SPIR.

\subsection{Spectroscopic investigation of crystalline spironolactone content}

Physical instability of amorphous solid dispersions during storage may lead to phase separation and crystallization. Crystalline traces can deteriorate the generally good dissolution of amorphous drugs which is a critical point from quality assurance viewpoint. In order to investigate the extent of dissolution reduction caused by crystalline SPIR in the case of tablets, various blends with different crystalline SPIR content were compressed into tablets and exposed to dissolution test.

It is discernible, that the more the crystalline SPIR content the worse the dissolution as it can be seen in Figure 10. Nonetheless, standard deviations were found to be really small in all cases. These results highlight that it is worth examining the crystallinity of the API during both the production and storage.

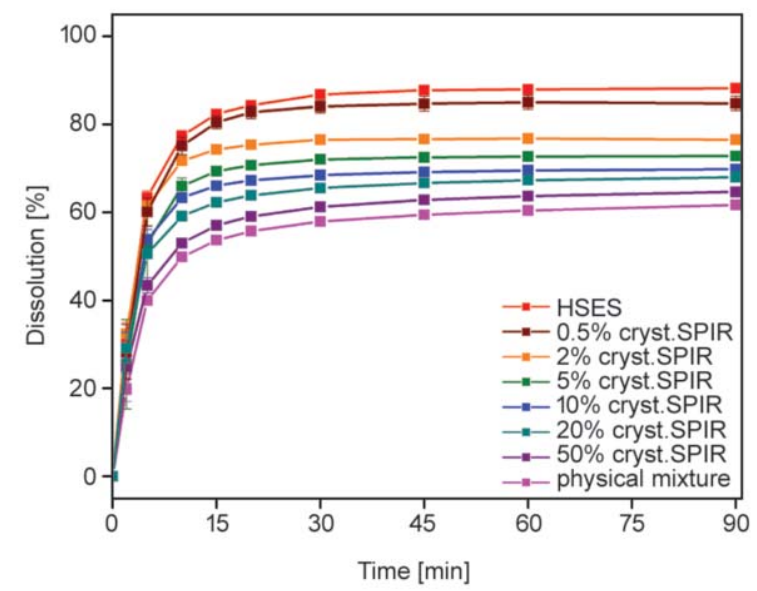

Figure 10. Dissolution of crystalline SPIR content tablets (900 mL $0.1 \mathrm{M} \mathrm{HCl}$ dissolution medium, $37 \pm 0.5^{\circ} \mathrm{C}, 100 \mathrm{rpm}$, paddle method, $50 \mathrm{mg}$ API content, $\mathrm{n}=3$ ).

\subsection{Results of the Raman and NIR spectroscopy}

As the presence of crystalline API can impair the dissolution, the fast detection of crystalline SPIR in the tablets during production by means of Raman and NIR spectroscopies might be very important for quality assurance. These techniques are non-destructive and can be applied without any sample preparation and in-line mode. Both tablets and powders were investigated by reflection mode and in the case of Raman spectroscopy moreover by transmission mode, too. Several combinations of spectral ranges and pre-processing methods were tested to obtain the most accurate regression (PLS) models. Hereinafter, only the models best fitting to each spectroscopic method and measuring mode are discussed.

Figure 11 and Figure 12 shows the coefficients of determination ( $R^{2}$ values), besides the predicted and measured concentrations for calibration and cross validation. In addition, limit of detection (LoD) and limit of quantificatiton (LoQ) values were calculated and presented in Table 3. In case of Raman models of powders, crystalline API cannot be detected, under $2.89 \%(\mathrm{LoD})$ and exact quantity of SPIR can be calculated over $8.75 \%$ (LoQ). If detecting lower crystallinities is still required, a calibration between 0 and $10 \%$ is necessary. The NIR spectroscopic model, compared to Raman results, determine the crystalline content of powders with 4.73 and $14.33 \%$ LoD/LoQ values, which are approximately 1.6 times higher than that of the Raman spectroscopy. Based on these results, more accurate detection can be achieved by Raman spectroscopy suggesting that it is more suitable for in-line analysis of crystallinity. Furthermore, it was also noticed that the calibration was more precise using powder samples. This is proven by the fact that the reflection Raman model of blends was found to be the most precise one.

Real time detection of crystalline traces by a non-destructive analytical technique such as NIR or Raman spectroscopy is of great importance regarding new pharmaceutical trend, continuous manufacturing

Table 3. Parameters of the models.

\begin{tabular}{|l|c|c|c|c|c|}
\hline \multirow{2}{*}{} & \multicolumn{2}{|c|}{ Results of NIR spectroscopy } & \multicolumn{3}{c|}{ Results of Raman spectroscopy } \\
\cline { 2 - 6 } & Blends & $\begin{array}{c}\text { Tablets } \\
\text { (reflection mode) }\end{array}$ & Blends & $\begin{array}{c}\text { Tablets } \\
\text { (reflection mode) }\end{array}$ & $\begin{array}{c}\text { Tablets } \\
\text { (transmission mode) }\end{array}$ \\
\hline RMSEC & 1.40 & 2.23 & 0.98 & 1.04 & 2.16 \\
\hline RMSECV & 1.62 & 4.16 & 1.19 & 1.60 & 2.64 \\
\hline LoQ & 14.33 & 23.77 & 8.75 & 10.22 & 23.60 \\
\hline LoD & 4.73 & 7.84 & 2.89 & 3.37 & 7.79 \\
\hline
\end{tabular}



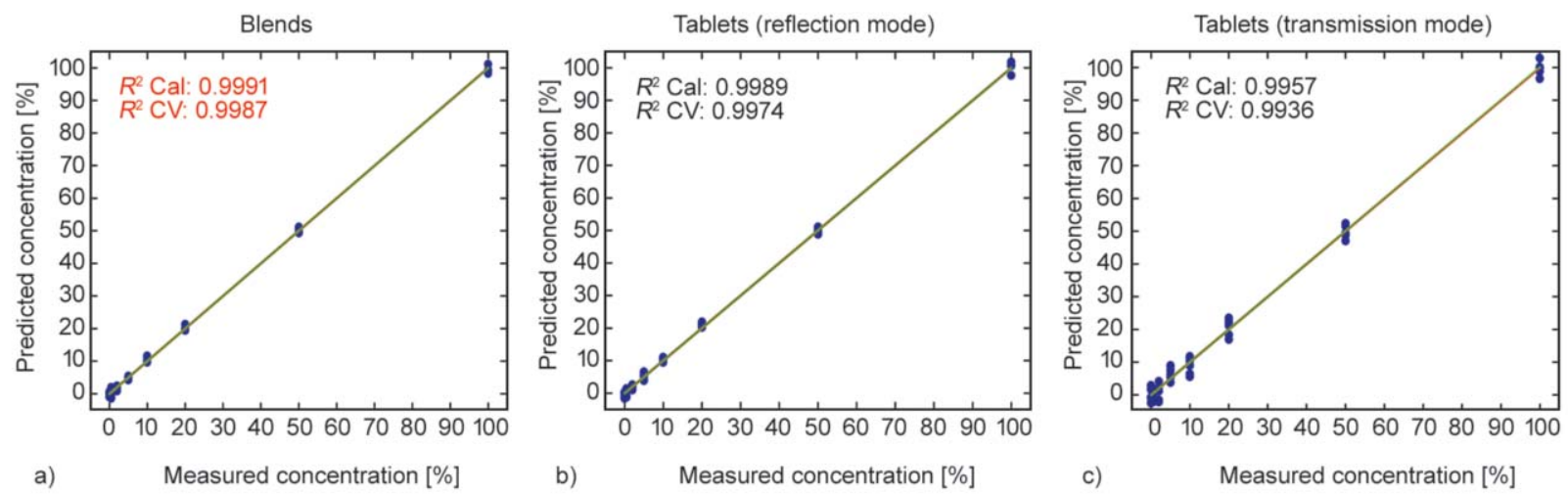

Figure 11. Predicted and measured data of Raman experiments. Reflection mode and transmission mode were used for measuring tablets $(b, c)$. Blends were investigated only in reflection mode (a).
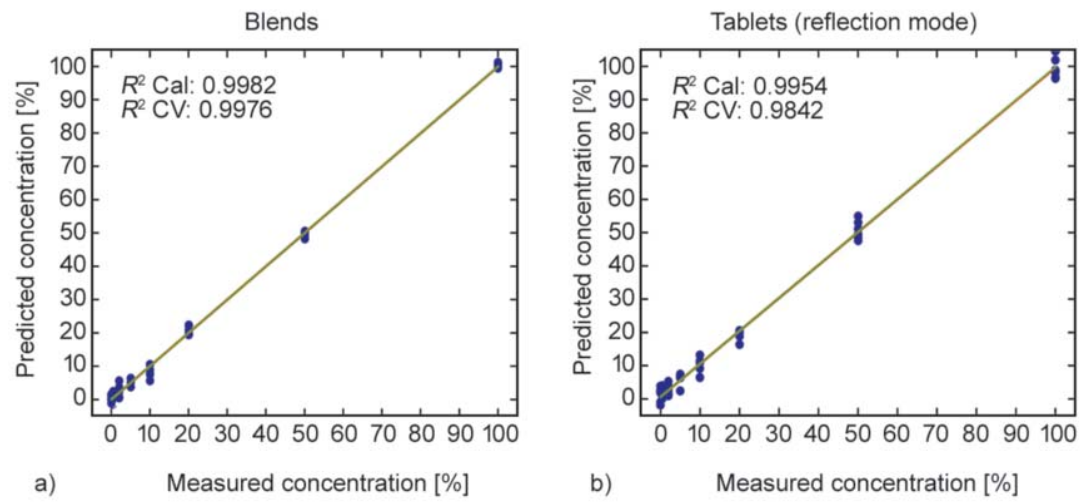

Figure 12. Predicted and measured concentration during NIR measurements in case of blends (a) and tablets (b).

[60]. A further goal of the future is to enhance the prediction power of the models and to correlate NIR and Raman spectra with the dissolution and achieve real-time release testing.

\subsection{Prediction of dissolution profile based on non-destructive measurement}

Dissolution results of the above mentioned calibration samples were served as the basis of the prediction. In the first step, dissolution values at 90 minutes were depicted as a function of the investigated SPIR contents (Figure 13). Based on this graph, a well-fitting power function relationship can be observed between the dissolution percentage and the crystalline API content. Similarly to the Figure 13, power functions can be fitted on the dissolution percentages versus crystalline drug content at all the dissolution sampling time points (Table 4). Utilizing these equations and the spectroscopically measured value of crystalline drug content dissolution percentages at the given time points can be calculated.

In a consequence, the described method seems to be appropriate to estimate the dissolution profiles of the tablets based on crystalline drug content determination using non-destructive spectroscopy (Raman or NIR) which can be considered as a real-time release testing method.

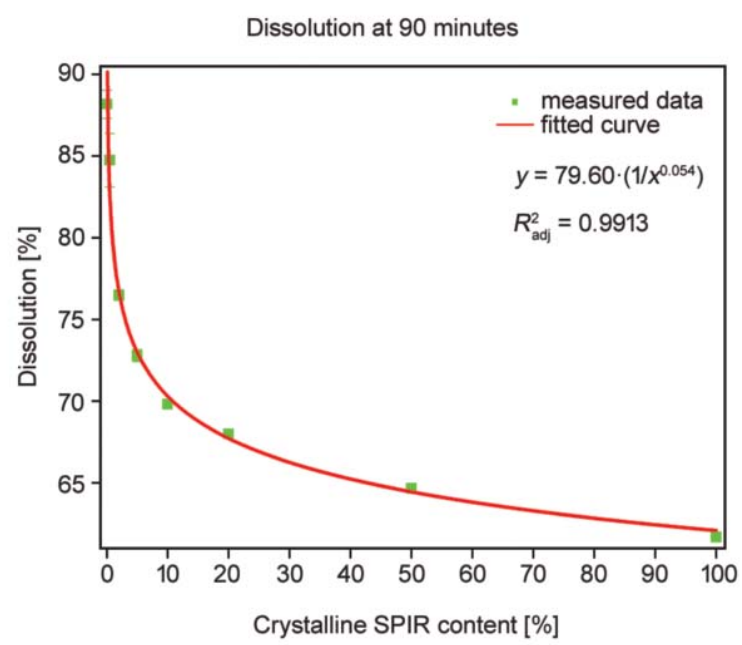

Figure 13. Dissolution values at 90 minutes as a function of crystalline API content $(900 \mathrm{~mL} 0.1 \mathrm{M} \mathrm{HCl}$ dissolution medium, $37 \pm 0.5^{\circ} \mathrm{C}, 100 \mathrm{rpm}$, paddle method, $50 \mathrm{mg}$ API content). 
Table 4. Fitted equations and the adjusted coefficient of determination $\left(R_{\text {adj }}^{2}\right)$.

\begin{tabular}{|c|c|c|}
\hline $\begin{array}{c}\text { Time } \\
{[\mathrm{min}]}\end{array}$ & Equation & $\boldsymbol{R}_{\text {adj }}^{\mathbf{2}}$ \\
\hline 5 & $y=65.503 \cdot \frac{1}{x^{0.099}}$ & 0.9538 \\
\hline 10 & $y=76.114 \cdot \frac{1}{x^{0.087}}$ & 0.98232 \\
\hline 15 & $y=78.652 \cdot \frac{1}{x^{0.080}}$ & 0.9934 \\
\hline 20 & $y=79.841 \cdot \frac{1}{x^{0.075}}$ & 0.99534 \\
\hline 30 & $y=80.5629 \cdot \frac{1}{x^{0.0705}}$ & 0.99806 \\
\hline 45 & $y=80.374 \cdot \frac{1}{x^{0.0636}}$ & 0.99543 \\
\hline 60 & $y=80.11 \cdot \frac{1}{x^{0.060}}$ & 0.9964 \\
\hline 90 & $y=79.606 \cdot \frac{1}{x^{0.0540}}$ & 0.9913 \\
\hline
\end{tabular}

\section{Conclusions}

In the present work, SPIR containing electrospun amorphous solid dispersions with different polymer matrices and drug loadings were characterized. According to the characterization methods applied (DSC, XRPD, SEM), all electrospun products were completely amorphous, and the micro-sized fibres were beneficial in terms of dissolution enhancement. PVPVA64 with $40 \%$ drug loading was found to be the most suitable composition for improved dissolution and scale-up using high speed electrospinning (HSES). HSES enables practical utilization of ASDs by preparing fibres more productively, and a continuous production of electrospun fibre-based tablets seems to be also doable. This latter statement is based on the feasible continuous collection, grinding, and precise feeding of the fibrous ASD.

Both NIR and Raman spectroscopies are suitable to detect crystalline SPIR in the prepared fibres and subsequent tablets. The Raman technique and reflection mode were found to be superior to NIR and transmission mode, respectively. Narrow calibration ranges (for instance, $0-10 \%$ ) would certainly give more accurate models, hence real-time process and quality control could be realized with these non-destructive analytical tools.
The obtained results confirm that scaled-up production of electrospun ASDs followed by continuous collection and continuous downstream processing (grinding, feeding, tableting) coupled with real-time process analytical technologies (PATs) is feasible. This way, not only the advantageous quality attributes of the electrospun ASDs but also the reliability of PAT controlled continuous production can be realized.

\section{Acknowledgements}

This project was supported by the New Széchenyi Plan (project ID: TÁMOP-4.2.1/B-09/1/KMR-2010-0002), OTKA grant KH 124541, PD 116122, PD-121051, GINOP-2.1.115-2015-00541, ÚNKP-17-4-I \& ÚNKP-17-4-II New National Excellence Program of the Ministry of Human Capacities, BME-KKP and the János Bolyai Research Scholarship of the Hungarian Academy of Sciences. This work was supported by the National Research, Development and Innovation Fund of Hungary in the frame of FIEK_16-1-2016-0007 (Higher Education and Industrial Cooperation Center) project.

\section{References}

[1] Amidon G. L., Lennernäs H., Shah V. P., Crison J. R.: A theoretical basis for a biopharmaceutic drug classification: The correlation of in vitro drug product dissolution and in vivo bioavailability. Pharmaceutical Research, 12, 413-420 (1995). https://doi.org/10.1023/A:1016212804288

[2] Savjani K. T., Gajjar A. K., Savjani J. K.: Drug solubility: Importance and enhancement techniques. ISRN Pharmaceutics, 2012, 195727/1-195727/10 (2012). https://doi.org/10.5402/2012/195727

[3] Singh A., van Humbeeck J., van den Mooter G.: A new twist in the old story-can compression induce mixing of phase separated solid dispersions? A case study of spray-dried miconazole-PVP VA64 solid dispersions. Pharmaceutical Research, 31, 3191-3200 (2014). https://doi.org/10.1007/s11095-014-1411-6

[4] Vasconcelos T., Sarmento B., Costa P.: Solid dispersions as strategy to improve oral bioavailability of poor water soluble drugs. Drug Discovery Today, 12, 1068-1075 (2007).

https://doi.org/10.1016/j.drudis.2007.09.005

[5] He Y., Ho C.: Amorphous solid dispersions: Utilization and challenges in drug discovery and development. Journal of Pharmaceutical Sciences, 104, 3237-3258 (2015). https://doi.org/10.1002/jps.24541

[6] Vo C. L-N., Park C., Lee B-J.: Current trends and future perspectives of solid dispersions containing poorly water-soluble drugs. European Journal of Pharmaceutics and Biopharmaceutics, 85, 799-813 (2013). https://doi.org/10.1016/j.ejpb.2013.09.007 
[7] Nagy Zs. K., Balogh A., Vajna B., Farkas A., Patyi G., Kramarics Á., Marosi Gy.: Comparison of electrospun and extruded Soluplus ${ }^{\circledR}$-based solid dosage forms of improved dissolution. Journal of Pharmaceutical Sciences, 101, 322-332 (2012).

https://doi.org/10.1002/jps.22731

[8] Verreck G., Chun I., Peeters J., Rosenblatt J., Brewster M. E.: Preparation and characterization of nanofibers containing amorphous drug dispersions generated by electrostatic spinning. Pharmaceutical Research, 20, 810-817 (2003).

https://doi.org/10.1023/A:1023450006281

[9] Williams G. R., Chatterton N. P., Nazir T., Yu D-G., Zhu L-M., Branford-White C. J.: Electrospun nanofibers in drug delivery: Recent developments and perspectives. Therapeutic Delivery, 3, 515-533 (2012).

https://doi.org/10.4155/tde.12.17

[10] Nagy Zs. K., Nyúl K., Wagner I., Molnár K., Marosi Gy.: Electrospun water soluble polymer mat for ultrafast release of Donepezil HCl. Express Polymer Letters, 4, 763-772 (2010).

https://doi.org/10.3144/expresspolymlett.2010.92

[11] Nagy Zs. K., Wagner I., Suhajda Á., Tobak T., Harasztos A. H., Vigh T., Sóti P., Pataki H., Molnár K., Marosi Gy.: Nanofibrous solid dosage form of living bacteria prepared by electrospinning. Express Polymer Letters, 8, 352-361 (2014).

https://doi.org/10.3144/expresspolymlett.2014.39

[12] Vigh T., Démuth B., Balogh A., Galata D. L., Van Assche I., Mackie C., Vialpando M., Van Hove B., Psathas P., Borbás E., Pataki H., Boeykens P., Marosi Gy., Verreck G., Nagy Zs. K.: Oral bioavailability enhancement of flubendazole by developing nanofibrous solid dosage forms. Drug Development and Industrial Pharmacy, 43, 1126-1133 (2017).

https://doi.org/10.1080/03639045.2017.1298121

[13] Paaver U., Heinämäki J., Laidmäe I., Lust A., Kozlova J., Sillaste E., Kirsimäe K., Veski P., Kogermann K.: Electrospun nanofibers as a potential controlled-release solid dispersion system for poorly water-soluble drugs. International Journal of Pharmaceutics, 479, 252-260 (2015).

https://doi.org/10.1016/j.ijpharm.2014.12.024

[14] Lopez F. L., Shearman G. C., Gaisford S., Williams G. R.: Amorphous formulations of indomethacin and griseofulvin prepared by electrospinning. Molecular Pharmaceutics, 11, 4327-4338 (2014).

https://doi.org/10.1021/mp500391y

[15] Yu D-G., Gao L-D., White K., Branford-White C., Lu W-Y., Zhu L-M.: Multicomponent amorphous nanofibers electrospun from hot aqueous solutions of a poorly soluble drug. Pharmaceutical Research, 27, 2466-2477 (2010).

https://doi.org/10.1007/s11095-010-0239-y

[16] Sun K., Li Z.: Preparations, properties and applications of chitosan based nanofibers fabricated by electrospinning. Express Polymer Letters, 5, 342-361 (2011). https://doi.org/10.3144/expresspolymlett.2011.34
[17] Zhuo H. T., Hu J. L., Chen S. J.: Coaxial electrospun polyurethane core-shell nanofibers for shape memory and antibacterial nanomaterials. Express Polymer Letters, 5, 182-187 (2011).

https://doi.org/10.3144/expresspolymlett.2011.16

[18] Zeng J., Xu X., Chen X., Liang Q., Bian X., Yang L., Jing X.: Biodegradable electrospun fibers for drug delivery. Journal of Controlled Release, 92, 227-231 (2003). https://doi.org/10.1016/S0168-3659(03)00372-9

[19] Xu Y., Li J-J., Yu D-G., Williams G. R., Yang J-H., Wang $\mathrm{X}$.: Influence of the drug distribution in electrospun gliadin fibers on drug-release behavior. European Journal of Pharmaceutical Sciences, 106, 422-430 (2017). https://doi.org/10.1016/j.ejps.2017.06.017

[20] Wang Q., Yu D-G., Zhang L-L., Liu X-K., Deng Y-C., Zhao M.: Electrospun hypromellose-based hydrophilic composites for rapid dissolution of poorly water-soluble drug. Carbohydrate Polymers, 174, 617-625 (2017). https://doi.org/10.1016/j.carbpol.2017.06.075

[21] Yu D-G., Li J-J., Zhang M., Williams G. R.: High-quality Janus nanofibers prepared using three-fluid electrospinning. Chemical Communications, 53, 4542-4545 (2017). https://doi.org/10.1039/c7cc01661a

[22] Yu D-G., Li X-Y., Wang X., Yang J-H., Bligh S. A., Williams G. R.: Nanofibers fabricated using triaxial electrospinning as zero order drug delivery systems. ACS Applied Materials and Interfaces, 7, 18891-18897 (2015). https://doi.org/10.1021/acsami.5b06007

[23] Rutledge G. C., Fridrikh S. V.: Formation of fibers by electrospinning. Advanced Drug Delivery Reviews, 59, 1384-1391 (2007). https://doi.org/10.1016/j.addr.2007.04.020

[24] Subbiah T., Bhat G. S., Tock R. W., Parameswaran S., Ramkumar S. S.: Electrospinning of nanofibers. Journal of Applied Polymer Science, 96, 557-569 (2005). https://doi.org/10.1002/app.21481

[25] Niu H., Lin T.: Fiber generators in needleless electrospinning. Journal of Nanomaterials, 2012, 725950/1725950/13 (2012).

https://doi.org/10.1155/2012/725950

[26] Molnár K., Nagy Zs. K.: Corona-electrospinning: Needleless method for high-throughput continuous nanofiber production. European Polymer Journal, 74, 279-286 (2016).

https://doi.org/10.1016/j.eurpolymj.2015.11.028

[27] Košt’áková E. K., Mészaros L., Maskova G., Blazkova L., Turcsan T., Lukas D.: Crystallinity of electrospun and centrifugal spun polycaprolactone fibers: A comparative study. Journal of Nanomaterials, 2017, 8952390/1-8952390/9 (2017).

https://doi.org/10.1155/2017/8952390

[28] Molnár K., Koštáková E., Mészáros L.: The effect of needleless electrospun nanofibrous interleaves on mechanical properties of carbon fabrics/epoxy laminates. Express Polymer Letters, 8, 62-72 (2013). https://doi.org/10.3144/expresspolymlett.2014.8 
[29] Zhou F-L., Gong R-H., Porat I.: Needle and needleless electrospinning for nanofibers. Journal of Applied Polymer Science, 115, 2591-2598 (2010).

https://doi.org/10.1002/app.31282

[30] Pokorny P., Košt’áková E., Sanetrnik F., Mikes P., Chvojka J., Kalous T., Bilek M., Pejchar K., Valtera J., Lukas D.: Effective AC needleless and collectorless electrospinning for yarn production. Physical Chemistry Chemical Physics, 16, 26816-26822 (2014). https://doi.org/10.1039/C4CP04346D

[31] Balogh A., Farkas B., Verreck G., Mensch J., Borbás E., Nagy B., Marosi Gy., Nagy Zs. K.: AC and DC electrospinning of hydroxypropylmethylcellulose with polyethylene oxides as secondary polymer for improved drug dissolution. International Journal of Pharmaceutics, 505, 159-166 (2016).

https://doi.org/10.1016/j.ijpharm.2016.03.024

[32] Balogh A., Farkas B., Domokos A., Farkas A., Démuth B., Borbás E., Nagy B., Marosi Gy., Nagy Zs. K.: Controlled-release solid dispersions of Eudragit ${ }^{\circledR}$ FS 100 and poorly soluble spironolactone prepared by electrospinning and melt extrusion. European Polymer Journal, 95, 406-417 (2017). https://doi.org/10.1016/j.eurpolymj.2017.08.032

[33] Nagy Zs. K., Balogh A., Démuth B., Pataki H., Vigh T., Szabó B., Molnár K., Schmidt B. T., Horák P., Marosi Gy., Verreck G., Van Assche I., Brewster M. E.: High speed electrospinning for scaled-up production of amorphous solid dispersion of itraconazole. International Journal of Pharmaceutics, 480, 137-142 (2015).

https://doi.org/10.1016/j.ijpharm.2015.01.025

[34] Buchholz S.: Future manufacturing approaches in the chemical and pharmaceutical industry. Chemical Engineering and Processing: Process Intensification, 49, 993-995 (2010).

https://doi.org/10.1016/j.cep.2010.08.010

[35] Poechlauer P., Manley J., Broxterman R., Gregertsen B., Ridemark M.: Continuous processing in the manufacture of active pharmaceutical ingredients and finished dosage forms: An industry perspective. Organic Process Research and Development, 16, 1586-1590 (2012). https://doi.org/10.1021/op300159y

[36] Balboni M. L.: Process analytical technology. Pharmaceutical Technology, 10, 54-66 (2003).

[37] Casian T., Reznek A., Vonica-Gligor A. L., Van Renterghem J., De Beer T., Tomuță I.: Development, validation and comparison of near infrared and Raman spectroscopic methods for fast characterization of tablets with amlodipine and valsartan. Talanta, 167, 333-343 (2017). https://doi.org/10.1016/j.talanta.2017.01.092

[38] Moes J. J., Ruijken M. M., Gout E., Frijlink H. W., Ugwoke M. I.: Application of process analytical technology in tablet process development using NIR spectroscopy: Blend uniformity, content uniformity and coating thickness measurements. International Journal of Pharmaceutics, 357, 108-118 (2008).

https://doi.org/10.1016/j.ijpharm.2008.01.062
[39] Porfire A., Rus L., Vonica A. L., Tomuta I.: High-throughput NIR-chemometric methods for determination of drug content and pharmaceutical properties of indapamide powder blends for tabletting. Journal of Pharmaceutical and Biomedical Analysis, 70, 301-309 (2012).

https://doi.org/10.1016/j.jpba.2012.07.026

[40] De Beer T., Vercruysse P., Burggraeve A., Quinten T., Ouyang J., Zhang X., Vervaet C., Remon J. P., Baeyens W.: In-line and real-time process monitoring of a freeze drying process using Raman and NIR spectroscopy as complementary process analytical technology (PAT) tools. Journal of Pharmaceutical Sciences, 98, 34303446 (2009).

https://doi.org/10.1002/jps.21633

[41] Nagy B., Farkas A., Gyürkés M., Komáromy-Hiller S., Démuth B., Szabó B., Nusser D., Borbás E., Marosi Gy., Nagy Zs. K.: In-line Raman spectroscopic monitoring and feedback control of a continuous twin-screw pharmaceutical powder blending and tableting process. International Journal of Pharmaceutics, 530, 21-29 (2017). https://doi.org/10.1016/j.ijpharm.2017.07.041

[42] Démuth B., Farkas A., Pataki H., Balogh A., Szabó B., Borbás E., Sóti P. L., Vigh T., Kiserdei É., Farkas B., Mensch J., Verreck G., Van Assche I., Marosi Gy., Nagy Zs. K.: Detailed stability investigation of amorphous solid dispersions prepared by single-needle and high speed electrospinning. International Journal of Pharmaceutics, 498, 234-244 (2016). https://doi.org/10.1016/j.ijpharm.2015.12.029

[43] Hitzer P., Bäuerle T., Drieschner T., Ostertag E., Paulsen K., van Lishaut H., Lorenz G., Rebner K.: Process analytical techniques for hot-melt extrusion and their application to amorphous solid dispersions. Analytical and Bioanalytical Chemistry, 409, 4321-4333 (2017). https://doi.org/10.1007/s00216-017-0292-z

[44] Lust A., Strachan C. J., Veski P., Aaltonen J., Heinämäki J., Yliruusi J., Kogermann K.: Amorphous solid dispersions of piroxicam and Soluplus ${ }^{\circledR}$ : Qualitative and quantitative analysis of piroxicam recrystallization during storage. International Journal of Pharmaceutics, 486, 306-314 (2015). https://doi.org/10.1016/j.ijpharm.2015.03.079

[45] Widjaja E., Kanaujia P., Lau G., Ng W. K., Garland M., Saal C., Hanefeld A., Fischbach M., Maio M., Tan R. B.: Detection of trace crystallinity in an amorphous system using Raman microscopy and chemometric analysis. European Journal of Pharmaceutical Sciences, 42, 45-54 (2011). https://doi.org/10.1016/j.ejps.2010.10.004

[46] Zidan A. S., Rahman Z., Sayeed V., Raw A., Yu L., Khan M. A.: Crystallinity evaluation of tacrolimus solid dispersions by chemometric analysis. International Journal of Pharmaceutics, 423, 341-350 (2012).

https://doi.org/10.1016/j.ijpharm.2011.11.003 
[47] Vigh T., Drávavölgyi G., Sóti P. L., Pataki H., Igricz T., Wagner I., Vajna B., Madarász J., Marosi Gy., Nagy Zs. K.: Predicting final product properties of melt extruded solid dispersions from process parameters using Raman spectrometry. Journal of Pharmaceutical and Biomedical Analysis, 98, 166-177 (2014).

https://doi.org/10.1016/j.jpba.2014.05.025

[48] Vigh T., Horváthová T., Balogh A., Sóti P. L., Drávavölgyi G., Nagy Zs. K., Marosi Gy.: Polymer-free and polyvinylpirrolidone-based electrospun solid dosage forms for drug dissolution enhancement. European Journal of Pharmaceutical Sciences, 49, 595-602 (2013). https://doi.org/10.1016/j.ejps.2013.04.034

[49] Hausner H.: Friction conditions in a mass of metal powder. Powder Metall, 13, 7-13 (1967).

[50] Carr R. L.: Evaluating flow properties of solids. Chemical Engineering, 72, 163-168 (1965).

[51] Nørgaard L., Saudland A., Wagner J., Nielsen J. P., Munck L., Engelsen S. B.: Interval partial least-squares regression (iPLS): A comparative chemometric study with an example from near-infrared spectroscopy. Applied Spectroscopy, 54, 413-419 (2000).

https://doi.org/10.1366/0003702001949500

[52] Borbás E., Sinkó B. 1., Tsinman O., Tsinman K., Kiserdei É., Démuth B., Balogh A., Bodák B., Domokos A., Dargó G., Balogh Gy. T., Nagy Zs. K.: Investigation and mathematical description of the real driving force of passive transport of drug molecules from supersaturated solutions. Molecular Pharmaceutics, 13, 3816-3826 (2016). https://doi.org/10.1021/acs.molpharmaceut.6b00613

[53] Jiang Y-N., Mo H-Y., Yu D-G.: Electrospun drug-loaded core-sheath $\mathrm{PVP} /$ zein nanofibers for biphasic drug release. International Journal of Pharmaceutics, 438, 232239 (2012).

https://doi.org/10.1016/j.ijpharm.2012.08.053
[54] Mit-uppatham C., Nithitanakul M., Supaphol P.: Ultrafine electrospun polyamide-6 fibers: Effect of solution conditions on morphology and average fiber diameter. Macromolecular Chemistry and Physics, 205, 23272338 (2004). https://doi.org/10.1002/macp.200400225

[55] Casasola R., Thomas N. L., Trybala A., Georgiadou S.: Electrospun poly lactic acid (PLA) fibres: Effect of different solvent systems on fibre morphology and diameter. Polymer, 55, 4728-4737 (2014).

https://doi.org/10.1016/j.polymer.2014.06.032

[56] Balogh A., Farkas B., Faragó K., Farkas A., Wagner I., Van Assche I., Verreck G., Nagy Zs. K., Marosi Gy.: Melt-blown and electrospun drug-loaded polymer fiber mats for dissolution enhancement: A comparative study. Journal of Pharmaceutical Sciences, 104, 1767-1776 (2015). https://doi.org/10.1002/jps.24399

[57] Costa P.: An alternative method to the evaluation of similarity factor in dissolution testing. International Journal of Pharmaceutics, 220, 77-83 (2001). https://doi.org/10.1016/S0378-5173(01)00651-2

[58] Moore J. W.: Mathematical comparison of dissolution profiles. Pharmaceutical Technology, 20, 64-75 (1996).

[59] Démuth B., Farkas A., Szabó B., Balogh A., Nagy B., Vágó E., Vigh T., Tinke A. P., Kazsu Z., Demeter Á., Bertels J., Mensch J., Van Dijck A., Verreck G., Van Assche I., Marosi Gy., Nagy Zs. K.: Development and tableting of directly compressible powder from electrospun nanofibrous amorphous solid dispersion. Advanced Powder Technology, 28, 1554-1563 (2017). https://doi.org/10.1016/j.apt.2017.03.026

[60] Lee S. L., O’Connor T. F., Yang X., Cruz C. N., Chatterjee S., Madurawe R. D., Moore C. M., Yu L. X., Woodcock J.: Modernizing pharmaceutical manufacturing: From batch to continuous production. Journal of Pharmaceutical Innovation, 10, 191-199 (2015).

https://doi.org/10.1007/s12247-015-9215-8 